\title{
Cooperative Transmit-Power Estimation under Wireless Fading
}

\author{
Murtaza Zafer \\ IBM T. J. Watson Research \\ 19 Skyline drive \\ Hawthorne, NY 10532 \\ mzafer@us.ibm.com
}

\author{
BongJun Ko \\ IBM T. J. Watson Research \\ 19 Skyline drive \\ Hawthorne, NY 10532 \\ bongjun_ko@us.ibm.com
}

\author{
Ivan Wang-Hei Ho \\ Department of Electrical and \\ Electronic Engineering \\ Imperial College London, UK \\ wh.ho@imperial.ac.uk
}

\begin{abstract}
We study blind estimation of transmission power of a node based on received power measurements obtained under wireless fading. Specifically, the setup consists of a set of monitors that measure the signal power received from the transmitter, and the goal is to utilize these measurements to estimate the transmission power in the absence of any prior knowledge of the transmitter's location or any statistical distribution of its power. Towards this end, we exploit spatial diversity in received-power measurements and cooperation among the multiple monitoring nodes; based on theoretical analysis we obtain the Maximum Likelihood (ML) estimate, derive fundamental geometrical insights and show that this estimate is asymptotically optimal. Finally, we provide numerical results comparing the performance of the estimators through simulations and on a data-set of field measurements.
\end{abstract}

\section{Categories and Subject Descriptors}

C.2.1 [Computer-Communication Networks]: Wireless communication - power estimation, localization

\section{General Terms}

Theory, Design, Performance, Algorithms

\section{Keywords}

Cooperative monitoring, Power estimation, Maximum likelihood, Wireless, Sensor network, Mobile ad-hoc network

\section{INTRODUCTION}

Estimation of transmission power has far-reaching applicability in event detection in sensor networks, monitoring of wireless networks, user detection in cognitive radio systems and power-aware design of mobile ad-hoc networks. In sensor networks, consider for example, a region where the sensor nodes are deployed to detect transient events, such

Permission to make digital or hard copies of all or part of this work for personal or classroom use is granted without fee provided that copies are not made or distributed for profit or commercial advantage and that copies bear this notice and the full citation on the first page. To copy otherwise, to republish, to post on servers or to redistribute to lists, requires prior specific permission and/or a fee.

MobiHoc'08, May 26-30, 2008, Hong Kong SAR, China.

Copyright 2008 ACM 978-1-60558-083-9/08/05 ...\$5.00. as an explosion. Since each sensor node can only measure the received power at its location, they would need to combine these measurements to estimate the power emitted at the source, which can then be utilized to make an event decision. Naturally, a priori, the location of the event is unknown and assuming any statistical prior knowledge might not be possible.

In wireless networks, the transmission power used by the nodes has ramifications in the operation of the entire network [1]. One important component in ensuring a proper and desired operation of such networks is monitoring the transmission power to detect abnormal or malicious behavior of nodes that can cause excessive signal interference. In such situations, monitors can be deployed as part of the regular nodes or as special nodes to estimate and monitor the transmission power behavior of other nodes. Power estimation may also be used to support power-aware operations in wireless networks such as transmission power control, medium-access control, routing etc., when correct transmission power information of the nodes is not readily available, either due to mis-configuration or device heterogeneity (e.g., lack or incompatible methods of transmit power indication). Transmission power estimation also has applicability in cognitive radio scenarios where secondary users need to detect the presence of primary users in their surroundings to avoid causing interference [8].

In this paper, we study "blind" estimation of transmission power based on received-power measurements at multiple $c o$ operative monitoring/sensor nodes. By blind, we mean that we do not assume any prior knowledge, statistical or otherwise, of the location of the transmitter or its transmit power. A key contribution of our work is to show that utilizing the spatial diversity in received-power measurements at multiple monitors, we can construct efficient blind estimators. More specifically the results are as follows - we obtain theoretical results for the Maximum Likelihood (ML) estimation under wireless Lognormal signal fading, and draw geometrical conclusions from the analysis; the geometric pictures help elucidate the problem and spell out various interesting and surprising insights. Furthermore, we show analytically that as the number of monitors increases the obtained estimate converges to the actual transmit power. As part of the numerical analysis, we present a simplified algorithm based on simple pairwise cooperation among the monitors that is amenable to a distributed implementation, and study the performance of various schemes through simulations and on a data-set of field measurements.

The power estimation problem that we consider is fairly 
unique from much of the research work in wireless and sensor networks, and its significant complexity arises from the fact that neither the transmit-power nor the transmitter location is known a-priori. In related work in sensor networks, cooperative node localization has been extensively studied [2-5], wherein, the goal is to locate the positions of the nodes in the network. Here, the nodes transmit with known power and compute pair-wise distances based on the received signal strength; these inter-distances are then combined together to locate the nodes. While this body of work is mainly concerned with node localization, our focus is on estimating the transmission power with the additional absence of location information. Though as a consequence of our analysis, we also provide the location estimate of the transmitter; furthermore, we draw the following insightful observation applicable to the setting of node localization (Corollary III.3): In the absence of transmission power information, and even under no wireless fading, the location of a transmitter cannot be uniquely determined if the monitors (even infinite number of them) lie on an arc of a circle or a straight line that does not pass through the actual transmitter location.

In other areas related to wireless network monitoring, there have been studies on Markov model based data fusion with the goal of estimating the sensor field $[6,7]$; however, our work is different and more specialized to power estimation under Lognormal fading. The power estimation problem that we consider also significantly differs from powercontrol problems studied in the literature on wireless and cellular networks [9-11]. The latter is a coordinated effort between the base station and the wireless node to reduce interference, while in our case there is no coordination assumed and the monitors independently measure the received signal strengths to estimate the transmit power.

In preliminary work in [12], we studied the geometric aspects of cooperative power estimation with up to three monitors under non-fading and deterministic signal propagation model. This paper provides a more thorough investigation of the problem under wireless fading, giving a formal estimator design, geometrical insights, asymptotic optimality and numerical results based on a data-set of field measurements.

\section{SYSTEM MODEL}

We consider a wireless network consisting of $N$ "monitors", where each monitor represents a wireless-node in a MANET environment or a sensor-node in a sensor network. Let $\mathcal{T}$ be the set of "transmitters" whose transmit-power is to be estimated; a transmitter refers to a physical node in a MANET environment or an event location in a sensor network field. We assume that the monitors can distinguish the signals of various transmitters; for example, in a sensor network, assuming that events occur at distinct times the power received from the events is easily distinguishable in time. In wireless networks with multiple transmitters, we consider situations where device identifiers of the transmitters are available through standard MAC, IP, or higher layer protocols (but the transmit power information is unknown or can be incorrect due to mis-configuration, incompatibility, or even malicious behavior). For our purposes, it therefore suffices to focus the mathematical analysis on the canonical single-transmitter multiple-monitor scenario.

Let $P$ denote the (unknown) transmit power and $d_{i}$ be the distance between the transmitter and monitor node $i(i=$ $1, \ldots, N)$. A priori, the value of $P$ and the location of the transmitter are unknown; hence, the distances $\left\{d_{i}\right\}_{i=1}^{N}$ are unknown. Furthermore, we do not make any assumptions about the statistical characteristics (e.g., mean, variance, distribution, etc.) of the transmission power $P$ and the location of the transmitter, since assigning any a-priori distributions would not be meaningful. Let $P_{i}$ denote the received power level at node $i$; this quantity is a random variable due to wireless fading. The uppercase notation $P_{i}$ will denote the random variable while the lowercase $p_{i}$ will denote a specific measured value. An implicit practical assumption throughout is that $p_{i}>R_{t h}$, where $R_{t h}$ is a positive constant for the minimum power level required to receive a signal.

For the signal propagation model, we consider the stochastic Lognormal fading model $[1,13]$. Let $d_{0}$ be some reference distance from the transmitter and $p_{0}$ be the received power level at that distance. The received power at node $i$ at distance $d_{i}$ from the transmitter is then given $\mathrm{as}^{1}$,

$$
\ln \left(\frac{P_{i}}{p_{0}}\right)=-\alpha \ln \left(\frac{d_{i}}{d_{0}}\right)+W_{i}
$$

where $\alpha>1$ is the path-loss exponent and $W_{i} \sim \mathcal{N}\left(0, \sigma^{2}\right)$ is an independent zero mean Gaussian random variable with variance $\sigma^{2}$. We note that the ML estimator presented in the later sections does not require the value of $\sigma$ to be known, however, it requires the value of $\alpha$. In practice, $\alpha \in[2,4]$ depending on the environment and can be estimated for the monitoring field by the monitors themselves through measurements of signal strength between them.

Rewriting (1), we get,

$$
\begin{aligned}
\ln P_{i} & =\ln \left(d_{i}^{-\alpha}\left(p_{0} d_{0}^{\alpha}\right)\right)+W_{i} \\
& =\ln (k P)+\ln \left(d_{i}^{-\alpha}\right)+W_{i}
\end{aligned}
$$

where, assuming $d_{0}$ is close to the transmitter, $\left(p_{0} d_{0}^{\alpha}\right)$ is proportional to the transmit power $P$ and is taken as $p_{0} d_{0}^{\alpha}=$ $k P$. Note that by taking $W_{i}=0$, the above model reduces to the deterministic power-law propagation model [1]. The justification for the above propagation model is as follows. First, since we are concerned with average signal power level, we consider large-scale fading model as opposed to shortterm fading which is used primarily for analysis at the databit level (eg. Rayleigh and Rician fading). Lognormal fading model is a widely studied long-term fading model and although simplistic in nature, it provides a good approximation for practical fading scenarios [14]. Additionally, it is also a tractable model which lends itself to analysis and provides fundamental insights for the estimation problem.

Now given only individual measurements and since the distance to the transmitter is unknown, each monitor node $i$ can make a simple guess that the transmit power $P \geq p_{i}$, assuming no fading happened. Furthermore, since we are concerned with measuring the power level of an event in a sensor field or monitoring misbehaving, ill-configured or malicious transmitters, monitor $i$ cannot directly know these quantities but must rely only on the received power measurements. In this work, we show that utilizing cooperation and joint power measurements $\left\{P_{i}\right\}_{i=1}^{N}$, the monitoring nodes can improve the estimation of transmit power $P$, thereby, usefully exploiting the spatial diversity in these measurements.

\footnotetext{
${ }^{1}$ Lognormal fading model is commonly defined in terms of $\log _{10}$ (dB scale) but for mathematical convenience we choose the natural logarithm.
} 


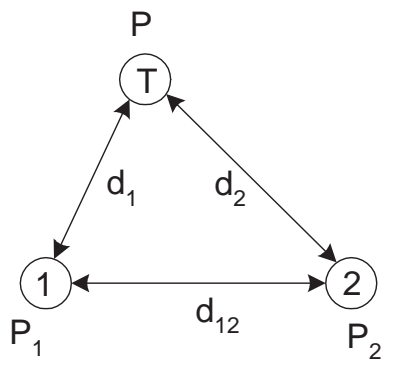

Figure 1: Two monitor scenario.

\section{TWO MONITOR SCENARIO}

We begin first with the two monitor case. Although a simple scenario, analyzing this setup serves two purposes. First, it provides fundamental insights and a valuable understanding of the estimation problem, and second, the estimator obtained for this case can be utilized to construct a "lightweight" distributed algorithm for transmit-power estimation under more general scenarios; this aspect is discussed later in Section 5.3.

Consider the setup shown in Figure 1, where we have a transmitter $T$ and two monitor nodes 1 and 2. As mentioned earlier, the transmit power $P$ and the transmitter location is unknown; hence, the distances $d_{1}$ and $d_{2}$ between the transmitter and nodes 1 and 2 respectively are unknown. Nodes 1 and 2 measure received-power levels $p_{1}$ and $p_{2}$ and can exchange this information among them. We assume that the distance $d_{12}$ between nodes 1 and 2 is known ${ }^{2}$. Using (3), we obtain,

$$
\begin{aligned}
& \ln \left(P_{1}\right)=\ln (k P)-\ln \left(d_{1}^{\alpha}\right)+W_{1} \\
& \ln \left(P_{2}\right)=\ln (k P)-\ln \left(d_{2}^{\alpha}\right)+W_{2}
\end{aligned}
$$

From the above equations, we see that the power estimation problem is a non-linear, Gaussian, non-random parameter estimation problem for which a valid, unbiased, minimumvariance estimate does not exist [15]. Therefore, in the rest of the work, we seek Maximum Likelihood (ML) estimates, and through analysis obtain valuable insights and discuss useful properties of these estimators.

Let $Z_{1}=\ln \left(P_{1}\right), Z_{2}=\ln \left(P_{2}\right)$ and $Z=\ln (P)$; we can then re-write (4) and (5) as,

$$
\begin{aligned}
& Z_{1}=Z+\left(\ln (k)-\ln \left(d_{1}^{\alpha}\right)\right)+W_{1} \\
& Z_{2}=Z+\left(\ln (k)-\ln \left(d_{2}^{\alpha}\right)\right)+W_{2}
\end{aligned}
$$

Let $\left(p_{1}, p_{2}\right)$ be the received-power, $\left(z_{1}, z_{2}\right)$ be the corresponding logarithm values and $\theta=\left(Z, d_{1}, d_{2}\right)$ denote the unknown parameter set. Let $f\left(z_{1}, z_{2} ; \theta\right)$ denote the joint probability density function, we then have,

$$
\begin{aligned}
& f\left(z_{1}, z_{2} ; \theta\right)=\prod_{i=1}^{2} f_{W_{i}}\left(z_{i}+\ln \left(d_{i}^{\alpha} / k\right)-Z\right) \\
& =\frac{1}{2 \pi \sigma^{2}} e^{-\frac{\left(z_{1}+\ln \left(d_{1}^{\alpha} / k\right)-Z\right)^{2}}{2 \sigma^{2}}} e^{-\frac{\left(z_{2}+\ln \left(d_{2}^{\alpha} / k\right)-Z\right)^{2}}{2 \sigma^{2}}}
\end{aligned}
$$

where the above follows since $W_{i} \sim \mathcal{N}\left(0, \sigma^{2}\right)$.

\footnotetext{
${ }^{2}$ Note that this assumption is weaker than having knowledge of the actual positions of the monitors; hence, the monitors can be arbitrarily placed in the network with known interdistance between them.
}

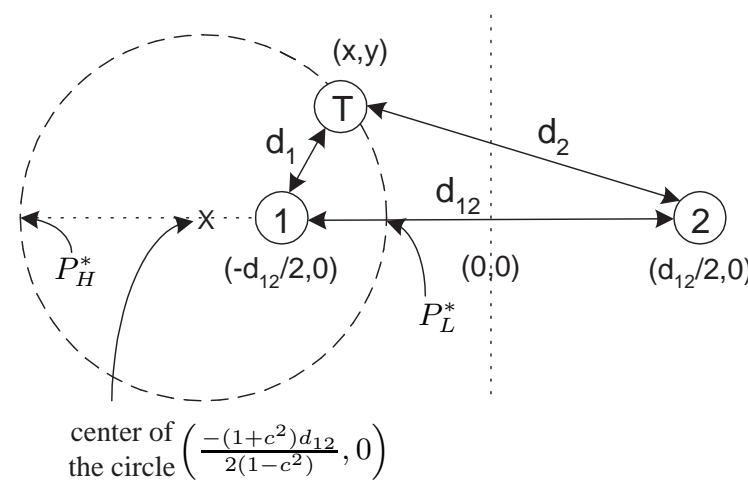

Figure 2: Schematic diagram showing that the ML estimate for two-monitor case corresponds to the transmitter located on a unique circle ( $c<1$ above).

Let $\Omega$ be the set of all admissible values for the parameter set $\theta$; note that $d_{1}$ and $d_{2}$ are not arbitrary as they must satisfy the triangle inequality. In Theorem I below, we give the Maximum Likelihood (ML) estimate which corresponds to those values of $\theta$ that maximize the chance of measuring $\left(z_{1}, z_{2}\right)$ at the two monitors respectively.

Theorem I. (ML estimate for two-monitor case) Let $\left(z_{1}, z_{2}\right)$ be the logarithm of the received-power at monitors 1 and 2 respectively, and $d_{12}$ be the distance between the monitors. Let $\theta^{*}=\left(Z^{*}, d_{1}^{*}, d_{2}^{*}\right)$ be the Maximum Likelihood $(M L)$ estimate; i.e.

$$
\theta^{*}=\arg \max _{\theta \in \Omega} f\left(z_{1}, z_{2} ; \theta\right)
$$

Then, the corresponding $Z^{*}$ is given as,

$$
Z_{L}^{*} \leq Z^{*} \leq Z_{H}^{*}
$$

where, for $z_{1} \geq z_{2}, Z_{L}^{*}=\ln \left(\frac{\left(d_{12}\right)^{\alpha}}{k\left(e^{-z_{1} / \alpha}+e^{-z_{2} / \alpha}\right)^{\alpha}}\right)$ and $Z_{H}^{*}=$ $\ln \left(\frac{\left(d_{12}\right)^{\alpha}}{k\left(e^{-z_{2} / \alpha}-e^{-z_{1} / \alpha}\right)^{\alpha}}\right)$; for $z_{2}>z_{1}, Z_{L}^{*}$ and $Z_{H}^{*}$ have the same expressions as before but interchanging $z_{1}$ and $z_{2}$.

REMARK 1. In simple terms, the above theorem states that there is a set of values of $\theta^{*}$ and corresponding $Z^{*}$ that maximize the likelihood of receiving measurements $\left(z_{1}, z_{2}\right)$. Furthermore, in the proof below, we point out an interesting geometric interpretation of the values $\left(d_{1}^{*}, d_{2}^{*}\right)$, summarized as follows - the ML estimate locus of the transmitter corresponds to a unique circle whose center and radius depends on the measured values $\left(z_{1}, z_{2}\right)$.

Proof. Without loss of generality, let node 1 be at location $\left(-d_{12} / 2,0\right)$ and node 2 at location $\left(d_{12} / 2,0\right)$. Consider the joint density function, $f\left(z_{1}, z_{2} ; \theta\right)$ which is a product of two functions $f_{W_{1}}(\cdot)$ and $f_{W_{2}}(\cdot)$ as given in (8). If we can find $\left(Z, d_{1}, d_{2}\right)$ that maximizes each function individually, then clearly, since each is a non-negative function the product is also maximized. Thus, for the maximization of $f\left(z_{1}, z_{2} ; \theta\right)$ we get two equations as follows,

$$
\begin{aligned}
& z_{1}-Z^{*}+\ln \left(\left(d_{1}^{*}\right)^{\alpha} / k\right)=0 \\
& z_{2}-Z^{*}+\ln \left(\left(d_{2}^{*}\right)^{\alpha} / k\right)=0
\end{aligned}
$$


Subtracting the above two equations gives,

$$
\frac{d_{1}^{*}}{d_{2}^{*}}=e^{\left(z_{2}-z_{1}\right) / \alpha}=c
$$

where $c$ denotes the quantity $e^{\left(z_{2}-z_{1}\right) / \alpha}$.

First, assume $z_{2}<z_{1}$ which implies $c<1$. Re-writing, $d_{1}^{*}=\sqrt{\left(x+d_{12} / 2\right)^{2}+y^{2}}, d_{2}^{*}=\sqrt{\left(x-d_{12} / 2\right)^{2}+y^{2}}$, equation (14) reduces to $\left(1-c^{2}\right) x^{2}+\left(1-c^{2}\right) y^{2}+\left(1+c^{2}\right) d_{12} x+$ $\left(1-c^{2}\right) d_{12}^{2} / 4=0$. This is an equation of a circle with center $\left(\frac{-\left(1+c^{2}\right) d_{12}}{2\left(1-c^{2}\right)}, 0\right)$ and radius $\frac{c d_{12}}{\left(1-c^{2}\right)}$ (as shown in Figure 2). From the circle, we get $\frac{c d_{12}}{1+c} \leq d_{1}^{*} \leq \frac{c d_{12}}{1-c}$; substituting this in (12) and simplifying gives, $Z_{L}^{*} \leq Z^{*} \leq Z_{H}^{*}$, where $Z_{L}^{*}$ and $Z_{H}^{*}$ are as stated in the theorem.

For the case $z_{2}>z_{1}$, the circle has center $\left(\frac{\left(1+c^{2}\right) d_{12}}{2\left(c^{2}-1\right)}, 0\right)$ and radius $\frac{c d_{12}}{\left(c^{2}-1\right)} ; Z_{L}^{*}$ is the same as before whereas $Z_{H}^{*}=$ $\ln \left(\frac{\left(d_{12}\right)^{\alpha}}{k\left(e^{-z_{1} / \alpha}-e^{-z_{2} / \alpha}\right)^{\alpha}}\right)$. Finally, for $z_{1}=z_{2}$, we get $d_{1}^{*} / d_{2}^{*}=$ 1 , which corresponds to the perpendicular bisector of the line joining nodes 1 and 2 . In this case $\frac{d_{12}}{2} \leq d_{1}^{*}<\infty$, thus, $Z_{H}^{*}=\infty$.

Let $P^{*}=e^{Z^{*}}$ denote the value of $Z^{*}$ in the power domain. Similarly, let $P_{L}^{*}=e^{Z_{L}^{*}}$ and $P_{H}^{*}=e^{Z_{H}^{*}}$. From (11) and using $p_{1}=e^{z_{1}}, p_{2}=e^{z_{2}}$, we have (for $z_{1}>z_{2}$ ), $P_{L}^{*}=$ $\frac{\left(d_{12}\right)^{\alpha}}{k\left(\left(1 / p_{1}\right)^{1 / \alpha}+\left(1 / p_{2}\right)^{1 / \alpha}\right)^{\alpha}}, P_{H}^{*}=\frac{\left(d_{12}\right)^{\alpha}}{k\left(\left(1 / p_{2}\right)^{1 / \alpha}-\left(1 / p_{2}\right)^{1 / \alpha}\right)^{\alpha}}$. Thus,

$$
P_{L}^{*} \leq P^{*} \leq P_{H}^{*}
$$

On the ML estimate circle, the locations which give values $P_{L}^{*}$ and $P_{H}^{*}$ respectively are depicted in Figure 2 .

From Theorem I several useful insights can be drawn as follows. First, note that the center of the (maximum likelihood) circle does not lie at either node 1 or node 2 . This is in contrast to "localization" problems, where for triangulation, circles are drawn with centers at the various probe locations $[2,3]$. Instead, here, for $z_{2}<z_{1}$, the center lies at point $\left(\frac{-\left(1+c^{2}\right) d_{12}}{2\left(1-c^{2}\right)}, 0\right)$ on the line joining the two monitors, as shown in Figure 2. Furthermore, the radius of this circle is dependent on the measured power levels $\left(p_{1}, p_{2}\right)$. As the measured $p_{2}$ value approaches $p_{1}$ the center moves further away from node 1 and the radius also increases; thus, the difference between $P_{H}^{*}$ and $P_{L}^{*}$ increases. When $p_{1}=p_{2}$ the maximum likelihood point could lie anywhere on the bisector which gives the widest range for $P^{*}$. For the case $p_{2}>p_{1}$, the geometric picture is simply the mirror image about the bisector line. Another observation from equations (12) and (13) is that $2 Z^{*}=\ln \left(e^{z_{1}} d_{1}^{* \alpha} / k\right)+\ln \left(e^{z_{2}} d_{2}^{* \alpha} / k\right)=$ $\ln \left(p_{1} d_{1}^{* \alpha} p_{2} d_{2}^{* \alpha} / k^{2}\right)$. From this, $P^{*}$ can be written as,

$$
P^{*}=\frac{1}{k} \sqrt{p_{1}\left(d_{1}^{*}\right)^{\alpha} p_{2}\left(d_{2}^{*}\right)^{\alpha}}
$$

Thus, expressed differently, $P^{*}$ is proportional to the geometric mean of $p_{1}\left(d_{1}^{*}\right)^{\alpha}$ and $p_{2}\left(d_{2}^{*}\right)^{\alpha}$, and this interpretation conforms with the generalized results for the multiple monitor case given in the next section.

As mentioned before, the ML estimate $P^{*}$ consists of an interval $\left[P_{L}^{*}, P_{H}^{*}\right]$ and one can in theory pick any point in this interval. In Section 5.3, as a part of performance analysis, we discuss this issue further and present a distributed estimator based on the concept of coverage area of the two monitors.

\section{MULTIPLE MONITORS SCENARIO}

In the previous section, we analyzed the ML estimate for the two-monitor scenario. We now generalize the setup to the multiple monitor case; specifically, consider the case of $N$ monitors $(i=1, \ldots, N)$ with received power measurements $\left\{P_{i}\right\}_{i=1}^{N}$. With multiple monitors there is clearly more diversity in measurements and one should be able to exploit this spatial diversity to improve the power estimation. Towards this goal, we first present the ML estimate, outline the various geometrical observations and finally show asymptotic optimality; i.e. we show that the power estimate converges to the actual transmit power (almost surely) in a random monitor deployment.

\subsection{Maximum Likelihood Estimate}

Let $\left(x_{i}, y_{i}\right)$ denote the location of monitor node $i$ which is assumed to be known. Let $(x, y)$ denote the unknown location of the transmitter and $P$ be its transmit power. Let $Z_{i}=\ln \left(P_{i}\right)$ and $Z=\ln (P)$; then using (3) we have,

$$
Z_{i}=Z+\left(\ln (k)-\ln \left(d_{i}^{\alpha}\right)\right)+W_{i}, i=1, \ldots, N
$$

where $d_{i}=\sqrt{\left(x-x_{i}\right)^{2}+\left(y-y_{i}\right)^{2}}$. Let $\hat{\theta}=(Z, x, y)$ denote the unknown parameter set, $\mathbf{z}=\left(z_{1}, \ldots, z_{N}\right)$ be a particular measured vector of $\left\{Z_{i}\right\}_{i=1}^{N}$ and $f(\mathbf{z} ; \hat{\theta})$ be the joint probability density function. Since $\left\{W_{i}\right\}_{i=1}^{N}$ are independent Gaussian random variables we have,

$$
\begin{aligned}
f(\mathbf{z} ; \hat{\theta}) & =\prod_{i=1}^{N} f_{W_{i}}\left(z_{i}+\ln \left(d_{i}^{\alpha} / k\right)-Z\right) \\
& =\prod_{i=1}^{N} \frac{1}{\sqrt{2 \pi \sigma^{2}}} e^{-\frac{\left(z_{i}+\ln \left(d_{i}^{\alpha} / k\right)-Z\right)^{2}}{2 \sigma^{2}}}
\end{aligned}
$$

The next theorem presents the ML estimate $\hat{\theta^{*}}$ that maximizes the likelihood of having measurements $\left(z_{1}, \ldots, z_{N}\right)$.

Theorem II. (ML estimate for multiple-monitor case) Let $\mathbf{z}=\left(z_{1}, \ldots, z_{N}\right)$ be the logarithm of the power received at monitors 1 through $N$ respectively. Let $\hat{\theta}^{*}=\left(Z^{*}, x^{*}, y^{*}\right)$ denote the Maximum Likelihood (ML) estimate of $\hat{\theta}$; i.e.

$$
\hat{\theta}^{*}=\arg \max _{\hat{\theta}} f(\mathbf{z} ; \hat{\theta})
$$

Then, $Z^{*}$ is given as,

$$
Z^{*}=\ln (1 / k)+\ln \left(\prod_{i=1}^{N} e^{z_{i}}\left(d_{i}^{*}\right)^{\alpha}\right)^{1 / N}
$$

where $d_{i}^{*}=\sqrt{\left(x^{*}-x_{i}\right)^{2}+\left(y^{*}-y_{i}\right)^{2}}$ and $\left(x^{*}, y^{*}\right)$ is given as,

$$
\left(x^{*}, y^{*}\right)=\arg \min _{(x, y)} \sum_{i=1}^{N}\left(\ln \left(e^{z_{i}} d_{i}^{\alpha}\right)-\frac{\sum_{j=1}^{N} \ln \left(e^{z_{j}} d_{j}^{\alpha}\right)}{N}\right)^{2}
$$

Proof. See Appendix A.

REMARK 2. The results of Theorem II can be understood as follows. Using $p_{i}=e^{z_{i}}$ and setting $P^{*}=e^{Z^{*}}$, equations (21) and (22) can be written as,

$$
P^{*}=\frac{1}{k}\left(\prod_{i=1}^{N} p_{i}\left(d_{i}^{*}\right)^{\alpha}\right)^{1 / N}
$$




$$
\left(x^{*}, y^{*}\right)=\arg \min _{(x, y)} \sum_{i=1}^{N}\left(\ln \left(p_{i} d_{i}^{\alpha}\right)-\frac{\sum_{j=1}^{N} \ln \left(p_{j} d_{j}^{\alpha}\right)}{N}\right)^{2}
$$

From the above two equations we see that the $M L$ estimate $P^{*}$ is proportional to the geometric mean of terms $\left\{p_{i}\left(d_{i}^{*}\right)^{\alpha}\right\}$ as given in (23), where $d_{i}^{*}$ is the distance between monitor $i$ and the estimated location of the transmitter $\left(x^{*}, y^{*}\right)$. This $M L$ estimate $\left(x^{*}, y^{*}\right)$ is that value of $(x, y)$ which achieves the minimum in (24). Note that the minimization quantity in (24) is proportional to the sample variance of $\left\{\ln \left(p_{i} d_{i}^{\alpha}\right)\right\}_{i=1}^{N}$. Thus, $\left(x^{*}, y^{*}\right)$ is the value that minimizes the sample variance of $\left\{\ln \left(p_{i} d_{i}^{\alpha}\right)\right\}$.

As noted above, to obtain the ML estimate $P^{*}$ we need to first compute $\left(x^{*}, y^{*}\right)$ through the minimization in (24). In full generality of the setup, a closed form solution to this optimization is intractable. However, the objective function in (24) is continuously differentiable with respect to $(x, y)$; hence, one can obtain the partial derivatives with respect to $x$ and $y$. Setting these to zero, one can enlist all the local minima (and stationary points) and pick those that achieve the lowest value of the objective function. Alternatively, one can also utilize standard numerical techniques to obtain the minimum point, as done for the numerical results later.

\subsection{Asymptotic Optimality}

Having obtained the transmission power estimate for the multiple monitor scenario, an interesting question that arises is how does the estimate perform as the number of monitors increases. Intuitively, with increasing number of measurements one would have more spatial diversity and this should improve the estimate. In this section, we corroborate this intuition analytically and show that the ML estimate asymptotically converges to the actual transmit power. To prove this result we proceed in steps and along the way develop interesting geometric insights into the problem.

Consider equation (23) and suppose for the moment that instead of using $d_{i}^{*}$ we use the real distance $d_{r, i}$ between the transmitter and monitor $i$, which is magically known. Let $P_{r}^{*}$ denote this estimate and $\left(P_{1}, \ldots, P_{N}\right)$ be the random vector denoting the received power at the $N$ monitors. We then have,

$$
P_{r}^{*}=\frac{1}{k}\left(\prod_{i=1}^{N} P_{i}\left(d_{r, i}\right)^{\alpha}\right)^{1 / N}
$$

Consider now the scenario as $N$ increases; i.e. we increase the number of monitors where each monitor measures the received power independently. The following lemma shows that as $N \rightarrow \infty, P_{r}^{*}$ converges to the actual transmit power $P$ of the transmitter.

Lemma 1. Consider $P_{r}^{*}$ given in (25), then almost surely,

$$
\lim _{N \rightarrow \infty} P_{r}^{*}=P
$$

Proof. See Appendix B.

From the above lemma we see that if the real location of the transmitter is known then the estimate in (23) with the real distances converges to the actual transmit power. Clearly however, in our case the location of the transmitter is unknown; hence, the real distances are unknown. Instead the ML estimate $\left(x^{*}, y^{*}\right)$ is obtained from the minimization

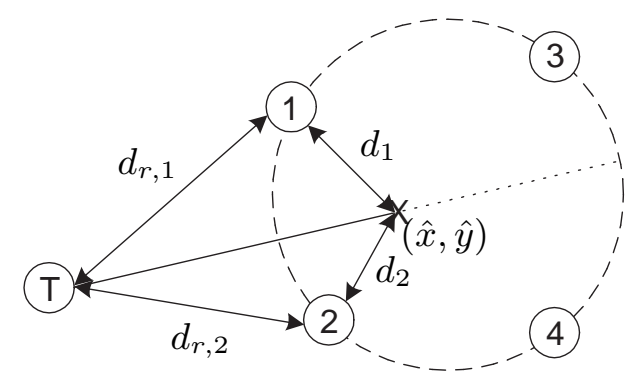

Figure 3: Schematic diagram showing the placement of monitors on a circular arc which gives two solutions - transmitter location and position marked $\mathrm{X}$.

in (24). We next show that under certain conditions as $N$ increases the optimization in (24) gives a unique solution which equals the actual location of the transmitter.

Before proceeding further, suppose for now that there is no fading, i.e. $W_{i}=0, \forall i$; analyzing this case will provide fundamental geometrical insights which will be utilized later. First, from (3) we see that under no fading, $p_{i}=k P / d_{r, i}^{\alpha}$, where as before, $d_{r, i}$ denotes the real distance between the transmitter and monitor $i$. Substituting this in (24) the minimization becomes,

$$
\begin{aligned}
& \min _{(x, y)} \sum_{i=1}^{N}\left(\ln \left(k P d_{i}^{\alpha} / d_{r, i}^{\alpha}\right)-\frac{\sum_{j=1}^{N} \ln \left(k P d_{j}^{\alpha} / d_{r, j}^{\alpha}\right)}{N}\right)^{2} \\
& \min _{(x, y)} \sum_{i=1}^{N}\left(\ln \left(d_{i}^{\alpha} / d_{r, i}^{\alpha}\right)-\frac{\sum_{j=1}^{N} \ln \left(d_{j}^{\alpha} / d_{r, j}^{\alpha}\right)}{N}\right)^{2}
\end{aligned}
$$

Note that the objective function above is non-negative. Clearly, $(x, y)$ equal to the actual location of the transmitter is a solution for (28) for which the minimized value equals zero (since $d_{i}=d_{r, i}$ in this case). The question then arises is whether there is another solution to the above minimization. Interestingly, the minimization in (28) has multiple solutions only for a specific placement of the monitors; this result is illustrated in the theorem below.

Theorem III. Consider the optimization given in (28). Let $\left(x^{*}, y^{*}\right)$ be the optimal solution, then $\left(x^{*}, y^{*}\right)$ is unique and equal to the actual location of the transmitter, except when the monitors are placed on an arc of a circle or a straight line that does not pass through the actual transmitter location.

Proof. Without loss of generality, let the real location of the transmitter be at the origin, then $d_{r, i}=\sqrt{x_{i}^{2}+y_{i}^{2}}$. Suppose that $(\hat{x}, \hat{y}) \neq(0,0)$ be another solution for (28). Clearly then, $(\hat{x}, \hat{y})$ must make the objective function in (28) equal to zero (since the minimum value achieved by the actual transmitter location equals zero). This will happen only if each term in the summation (28) is equal to zero, which requires,

$$
\frac{d_{i}}{d_{r, i}}=\beta,(\text { a constant }), \forall i=1, \ldots, N
$$

This can be further written as,

$$
\left(1-\beta^{2}\right) x_{i}^{2}+\left(1-\beta^{2}\right) y_{i}^{2}-2 x_{i} \hat{x}-2 y_{i} \hat{y}+\left(\hat{x}^{2}+\hat{y}^{2}\right)=0
$$

From the above equation we see that for $(\hat{x}, \hat{y})$ to be a solution of (28), the monitor locations $\left(x_{i}, y_{i}\right)$ must satisfy (30). 
But this is the equation of a circle for $\beta \neq 1$, and for $\beta=1$ it is a straight line that does not pass through the origin. Thus, stated differently, if the monitors are located on an arc of a circle or a straight line that does not pass through the actual transmitter location, we can find $(\hat{x}, \hat{y}) \neq(0,0)$ and $\beta>0$ such that (30) holds. For all other placements of the monitors, (29) cannot be satisfied; hence there cannot be more than one solution.

Corollary III.1. For the scenario of two monitors there are always multiple solutions for (28).

Proof. For any distinct placement of two monitors we can find a circular arc passing through the two points.

Corollary III.2. For the scenario of three monitors that do not lie on a straight line passing through the transmitter location there are always multiple solutions for (28).

Proof. Three monitors either form a triangle in which case there is a circle that passes through them, or they lie in a straight line. If this line does not pass through the transmitter location, then in either case Theorem III implies multiple solutions.

Figure 3 is a schematic diagram of the circular geometry of monitor placements which yields multiple solutions to (28).

An interesting outcome of Theorem III for the well-studied problem of node-localization is that even with infinite number of monitors and no fading, in the absence of transmit power information there are situations in which the transmitter location cannot be known with full accuracy.

Corollary III.3. Consider the problem of locating the transmitter position under power-law propagation model with no fading. In the absence of transmit power information, the transmitter location cannot be uniquely determined if the monitors lie on an arc of a circle or a straight line that does not pass through the actual transmitter location.

Proof. Under no fading, the propagation model in (3) can be written as $P_{i}=k P / d_{i}^{\alpha}$. Let $P$ be the actual (unknown) transmit power, then the received power is given as $P_{i}=k P / d_{r, i}^{\alpha}$, where $d_{r, i}$ is the actual distance between the transmitter and monitor $i$. Let $(\hat{x}, \hat{y})$ be some other location (with a corresponding transmit power $\hat{P}$ ) that also results in the same received powers; this happens if and only if,

$$
\hat{d}_{i} / d_{r, i}=\beta,(\text { a constant }), \forall i ; \text { and }, \quad \hat{P}=\beta^{\alpha} P
$$

From Theorem III we know that if the monitors are located on an arc of a circle or a straight line that does not pass through the actual transmitter location, then (31) can indeed be satisfied by another location different from the actual transmitter location. In such a situation, the two locations are indistinguishable based on received-power measurements.

We now return to the stochastic fading scenario and show asymptotic optimality of the ML estimate. The setup is as follows. Let $\Gamma$ denote a bounded region in $\mathbb{R}^{2}$ which encloses the origin. Without loss of generality, let the actual location of the transmitter be at the origin (which is of course unknown to the monitors). Let $N$ be the number of monitors. Place each monitor in $\Gamma$ independently according to the probability distribution $F_{X Y}(x, y)$; i.e. for monitor $i$ its location $\left(x_{i}, y_{i}\right) \in \Gamma$ is drawn from the distribution $F_{X Y}(x, y)$. Each monitor then independently makes received power measurement under Lognormal fading. Using these measurements and based on the result in Theorem II, we obtain the ML estimate of the transmit power. Let $P_{N}^{*}$ denote the estimated power, and $\left(x_{N}^{*}, y_{N}^{*}\right)$ be the ML estimate of the transmitter location obtained from the minimization in (24). The subscript $N$ in the notation denotes the number of monitors employed. We refer to this as the "Random Monitor Placement" scenario. Consider now a sequence of such setups indexed by $N$ where $N$ is monotonically increasing. Theorem IV below states that if the random distribution $F_{X Y}(x, y)$ does not place all the monitors on an arc of a circle or a straight line, the ML estimate given in Theorem II is asymptotically optimal; i.e. as $N$ goes to infinity it converges to the actual transmit power $P$.

Theorem IV. (Asymptotic optimality of ML estimate) Consider the random monitor placement scenario over a bounded region $\Gamma$. Let the monitor location $\left(x_{i}, y_{i}\right), \forall i$, be drawn independently from the distribution $F_{X Y}(x, y)$. Let $F_{X Y}(x, y)$ be such that,

1. $F_{X Y}(x, y)$ is not a distribution over an arc of a circle or a straight line in $\Gamma$.

2. $E[|L(\hat{x}, \hat{y})|]<\infty$ and $\operatorname{Var}(L(\hat{x}, \hat{y}))<\infty$, for all $(\hat{x}, \hat{y}) \in$ $\Gamma$, where $L(\hat{x}, \hat{y}) \triangleq \frac{\alpha}{2} \ln \left(\frac{(x-\hat{x})^{2}+(y-\hat{y})^{2}}{x^{2}+y^{2}}\right)$.

Let $P_{N}^{*}$ denote the $M L$ estimate of transmit power $P$ given by (23) for an $N$ monitor scenario. Then, almost surely,

$$
\lim _{N \rightarrow \infty} P_{N}^{*}=P
$$

Proof. See Appendix C.

\section{PERFORMANCE EVALUATION}

The ML estimate obtained in the previous section has the interesting property of asymptotic convergence; however, while the asymptotic result provides justification for this estimate, we would still like to quantify its performance for finite monitor scenarios. In this section, we study this performance through numerical analysis based on simulations and a data set of field measurements of received power.

\subsection{Performance Metrics}

Let $\mathcal{E}_{d B}$ denote the $\mathrm{dB}$ error of the estimate $P^{*}$ of a transmitter's actual (ground-truth) transmit power $P$, i.e., $\mathcal{E}_{d B}=$ $P_{d B m}^{*}-P_{d B m}, P_{d B m}^{*}=10 \log _{10} P^{*}$, and $P_{d B m}=10 \log _{10} P$. Given $K$ samples of transmit power $P$, we define the estimation error as the square-root of the mean square $\mathrm{dB}$ error, given as,

$$
\left(\mathbf{E}_{K}\left[\mathcal{E}_{d B}^{2}\right]\right)^{1 / 2}=\sqrt{\mathbf{E}_{K}\left[\left(P_{d B m}^{*}-P_{d B m}\right)^{2}\right]},
$$

where $\mathbf{E}_{K}$ denotes the ensemble average of $K$ observations.

We say that a transmission power estimator is effective under wireless lognormal fading with zero-mean and variance $\sigma_{d B}^{2}$, if $\mathbf{E}_{K}\left[\mathcal{E}_{d B}^{2}\right] \leq \sigma_{d B}^{2}{ }^{3}$. In our context of blind power estimation, an estimator being effective indicates that its error performance in terms of (33) is as good as the one by

\footnotetext{
${ }^{3} \sigma_{d B}^{2}$ is the variance of $W_{i}$ when equation (3) is expressed
} in $10 \log _{10}()$ scale rather than $\ln ()$; thus $\sigma=\sigma_{d B} \ln (10) / 10$. 
a single non-blind monitor that knows the exact distance to the transmitter. To elaborate, suppose a (non-cooperative) monitor knows the exact distance $d$ to a transmitter, and it estimates its transmit power $P$ based on the received power $p_{r}$. Knowing $d$ and $p_{r}$, this single monitor's estimate of $P$ is $P^{*}=(1 / k) p_{r} d^{\alpha}$, for which $\mathbf{E} \mathcal{E}_{d B}^{2}=\sigma_{d B}^{2}$. Hence, if a blind estimator is effective, its estimation error, $\left(\mathbf{E}_{K}\left[\mathcal{E}_{d B}^{2}\right]\right)^{1 / 2}$, is less than or equal to that of a single non-blind estimation. Our goal in this section is to illustrate via simulations that the cooperation of monitors can achieve effective blind estimation.

\subsection{Methodology}

We experiment with the following two estimation schemes:

- MLE-Coop: A centralized, cooperative ML power estimator given by (21) and (22).

- MLE-Pair: A decentralized extension of two-monitor ML estimator, which requires only the distances (not the locations) between pairing monitors to estimate the power. See Section 5.3 for further details.

We employ two different methods of MLE-Coop to find out the optimal solution of $\left(x^{*}, y^{*}\right)$ in $(22)$. The first method is to divide the entire monitored region into two-dimensional grid block, and choose the center position of the grid block that gives the minimum value of the optimization function in $(22)$ as the optimal $\left(x^{*}, y^{*}\right)$. The results in the later part of this section show that the inherent inaccuracy introduced by this quantization method has little impact on the overall accuracy of the power estimation. This is due to the fact that the unavoidable inaccuracy introduced by wireless fading neutralizes the effect of choosing slightly sub-optimal positions. The second approach is to use some optimization tool such as fminsearch in MATLAB. Despite potentially faster running time than grid-based search, such an optimization tool has its own drawback: they sometimes fall into local minima, and when they do, it can have drastically negative impact on the overall performance. We evaluate both approaches here, and distinguish them by denoting the former by MLE-Coop-grid (grid search) and the latter MLE-Coop-fmin (fminsearch).

Along with the above estimators, we also show results of an "ideal" power estimator, which is assumed to be able to obtain the precise location information of transmitters. This imaginary estimator calculates the maximum likelihood transmission power in (21) with correct distance information, and provides upper bounds of the performance of any blind estimator. We call it MLE-ideal.

The performance of the above power estimators are evaluated through two types of simulation using MATLAB. The first one is based on synthetic data set, in which a large volume of samples are generated for transmitters' and monitors' locations, transmit powers, and received powers under lognormal wireless fading. In the second setting, we utilize an empirical data-set of actual field measurements of received powers. We elaborate later the details of these evaluation processes in their respective sections.

\subsection{Distributed Pairwise Estimation}

MLE-Pair estimator is a distributed extension of twomonitor ML estimator presented in Section 3. Consider two

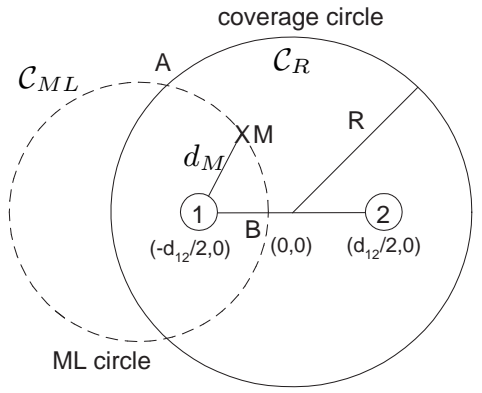

Figure 4: Schematic diagram showing the intersection of ML estimate circle and coverage circle.

monitors, and without loss of generality, let them be at locations $\left(-d_{12} / 2,0\right)$ and $\left(d_{12} / 2,0\right)$ respectively (see Figure 4). Recall from Section 3 that for the two monitor case, the estimated power $P^{*}$ consists of an interval based on the MLestimate circle, denoted by $\mathcal{C}_{M L}$ in the Figure. In principle one can pick any point in this interval; however, when $p_{1}$ and $p_{2}$ are close in value the size of the ML circle increases arbitrarily and this becomes meaningless from a practical perspective. To address this concern we introduce the concept of coverage area of the two monitors.

The coverage area is a circle (denoted by $\mathcal{C}_{R}$ in Figure 4 ) of radius $R$ centered at the origin. Essentially $\mathcal{C}_{R}$ defines the region that two monitors "cover" the transmitters within it to estimate their transmission powers. The rationale behind the coverage region is two fold: 1) Efficiency: by restricting the search space of potential position of the transmitter, the pair of monitors can avoid a pathological decision of choosing arbitrarily large estimate value, and 2) Divide-and-Conquer: the union of the coverage area of multiple pairs of monitors can cover a large part of the monitored region.

We now describe the estimation method that individual pairs of nodes employ. For any measured power values $p_{1}$ and $p_{2}$, we restrict the $\mathrm{ML}$ estimate circle to within $\mathcal{C}_{R}$, effectively limiting the likely position of the transmitter on the portion of ML circle $\mathcal{C}_{M L}$ that is contained within the area enclosed by $\mathcal{C}_{R}$. This portion corresponds to arc $A B$ in Figure 4 (the lower part of the arc gives the same values for the estimate $P^{*}$; hence it is not considered), where $A$ is the intersection point of $\mathcal{C}_{M L}$ with $\mathcal{C}_{R}$ and $B$ is the intersection point of $\mathcal{C}_{M L}$ and the line segment between the two monitors.

Within arc $A B$, we pick the mid-point $M$ on this arc and compute the power estimate based on the ML estimate at this point. Thus, for a pair of monitors $i$ and $j$, the estimate is given as, $P_{M L E-P a i r}^{*}(i, j)=\frac{1}{k} p_{1} d_{M}^{\alpha}$. The rationale behind this estimator is that restricting the transmitter's position on the arc $A B$, and, supposing all positions are equally likely, the mid point gives the mean location on arc $A B$. Finally, if $\mathcal{C}_{M L}$ is small enough and does not intersect $\mathcal{C}_{R}$, point $M$ is taken as the midpoint along the semi-circle of $\mathcal{C}_{M L}$.

Finally, the network-wide estimation $P_{M L E-P a i r}^{*}$ of transmit power is calculated by a localized, distributed protocol that enables the exchange of individual $P_{M L E-P a i r}^{*}(i, j)$ between neighboring pairs of nodes in a way similar to the routing protocol's operation. Having obtained other pairs' estimate values, the monitors can average those values to obtain $P_{M L E-P a i r}^{*}$. In our simulations, we simply use arithmetic mean to combine multiple local estimate. 


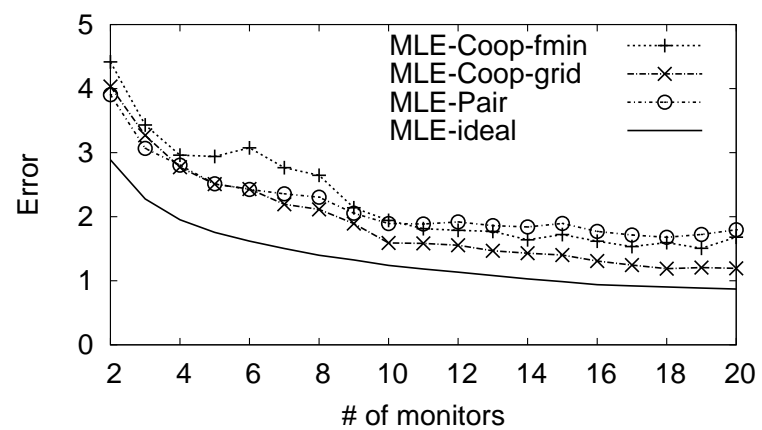

(a) Estimation error: $\left(\mathbf{E}_{K} \mathcal{E}_{d B}^{2}\right)^{1 / 2}$

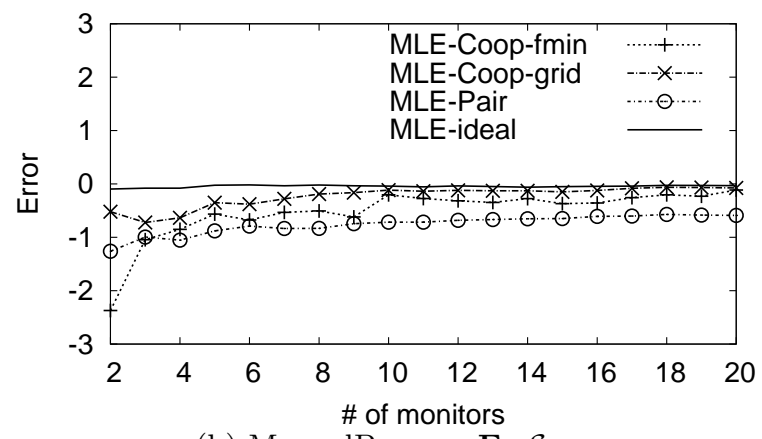

(b) Mean dB-error: $\mathbf{E}_{K} \mathcal{E}_{d B}$

Figure 5: Performance of estimators under random monitor/transmitter distribution: Monitors are placed uniformly at random.

\subsection{Evaluation using Synthetic Dataset}

In the synthetic simulation, $K$ transmitters and $N$ monitors are placed uniformly at random in a two-dimensional disk of radius $R$. The dB-transmit power, $P_{d B m}$ of each transmitter is selected uniformly at random from a range [min $\left.P_{d b}, \max P_{d b}\right]$. Then, each monitor's received power from a transmitter at distance $d$ is generated by an i.i.d. lognormal fading model given by (3). The MLE-Coop-grid estimator searches for the optimal point among $(20 \times 20) \times$ $\frac{\pi R^{2}}{(2 R)^{2}} \approx 314$ grid points. The MLE-pair estimation utilizes all pairs of $N$ monitors, thus averaging the results of twomonitor cooperative estimations by $N(N-1) / 2$ pairs, and each pair's coverage region is set to $R$ (regardless of the position of the pairs). For simulations, we used $R=40$, $K=1000$, and varied $N$ from 2 to 20 to observe the effect of increasing number of monitors. We used lognormal shadowing parameters, $\alpha=2.3$ and $\sigma_{d B}=3.92$, obtained from empirical measurement data used in Section 5.5

In Figure 5, we plot the estimation performance of all estimators under the above random monitor placement as a function of increasing number of monitors. Each point in the plot represents the performance value averaged over $K=1000$ estimation results performed by $N$ cooperating monitors in $x$-axis.

Figure 5(a) shows the estimation error $\left(\mathbf{E}_{K}\left[\mathcal{E}_{d B}^{2}\right]\right)^{1 / 2}$ given in (33). Recall from Section 5.1 that a blind estimator is asymptotically effective if the estimation error is less than or equal to $\sigma_{d B}=3.92$, and we can verify indeed all four estimators achieve below- $\sigma_{d B}$ performance when $N>2$, and generally continue to perform better as the number of coop-

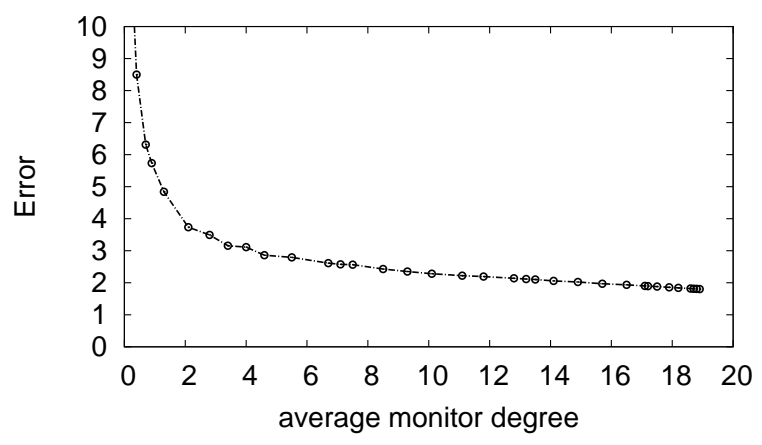

Figure 6: Scalability of MLE-pair under random monitor distribution: estimation error $\left(\left(\mathbf{E}_{K} \mathcal{E}_{d B}^{2}\right)^{1 / 2}\right)$ vs average monitor degree for pairwise estimation

erating monitors grows. This clearly highlights the benefit of nodes' cooperation in blind estimation.

Overall, MLE-Coop-grid performs the best among three (realizable) estimators, and quickly approaches the performance of the reference non-blind estimator, MLE-ideal. In the case of MLE-Coop-fmin, though it attempts to find the optimal solution more quickly, it comes at the expense of sometimes reaching local minima, the effect of which is shown in erratic performance when $N$ is between 4 to 8 .

Figure 5(b) additionally depicts the mean dB-error $\mathbf{E}_{K} \mathcal{E}_{d B}$, an indication of the bias of the estimators. While the twocentralized estimators gradually approach zero-mean as $N$ increases (except some erroneous cases of MLE-Coop-fmin), the convergence of the decentralized one (MLE-Pair) is relatively slow, indicating that taking the average of the pairwise estimations is biased slightly toward sub-mean value in this particular scenario. The relatively low performance of MLE-pair (compared to the centralized approaches) is expected since it is designed as a heuristic means for the scalability of estimation as a major concern.

We then further investigate the scalability of the MLEpair estimator. Here we place $K=1000$ transmitters and $N=20$ monitors uniformly at random within a disk of radius $R=20$. In this case, however, we control the pairwise connectivity by having the monitors make the pairing connection only if their distance is smaller than some $d_{\min }$. We observe the performance of MLE-pair estimator as a function of the number of monitor pairs (hence the average degree in the $x$-axis) by growing $d_{\min }$ until $d_{\min }$ becomes equal to $2 * R$, at which point the monitors are fully connected. Figure 6 shows the result. As seen in the figure, the estimation error of MLE-pair estimator quickly drops initially at low degree, and a knee is formed around the average degree 3, after which $\left(\mathbf{E}_{K} \mathcal{E}_{d B}^{2}\right)^{1 / 2}$ stabilizes below $\sigma_{d B}$. This experiment illustrates that the MLE-pair estimation can achieve effective performance even with the sparse connection between monitors.

\subsection{Evaluation using Empirical Dataset}

For the performance evaluation under a more realistic scenario, we use the power measurement data collected from a wireless sensor network [17]. This data set consists of 44 wireless sensor devices' measurement results, in which the received powers between all pairs of devices are measured. The actual transmit powers of all devices are fixed at -37.47 


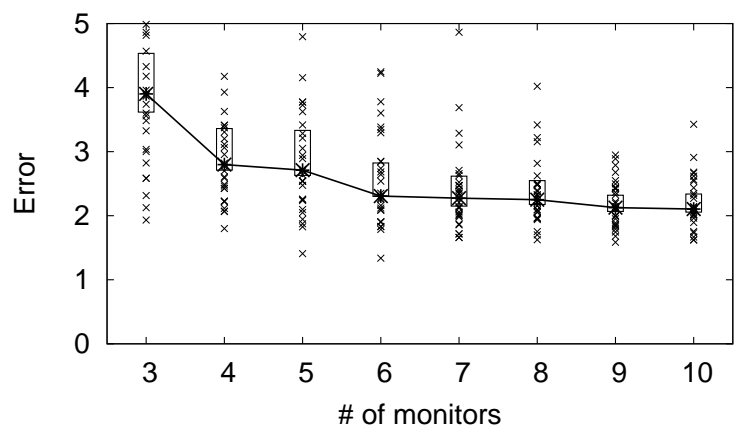

Figure 7: Estimation error $\left(\mathbf{E}_{K} \mathcal{E}_{d B}^{2}\right)^{1 / 2}$ of MLECoop-grid under field measurement data set: each point represents the output of an iteration, each with a randomly selected set of monitors.

$\mathrm{dBm}$, and hence the goal of the power estimation is to obtain values close to this fixed transmit power. The precise locations of the devices are also available in this data, which we only apply to MLE-ideal to compare the performance of our blind power estimator aginst an ideal (non-blind) power estimator.

A report on this field data [14] shows that the received power in the collected measurements can be fitted well to a lognormal fading model with path-loss exponent $\alpha=2.3$ and $\sigma_{d B}=3.92$.

We present in Figure 7 the estimation error of MLE-Coopgrid estimator. Each point in the plot represents $\left(\mathbf{E}_{K} \mathcal{E}_{d B}^{2}\right)^{1 / 2}$ value obtained from one simulation run with $N$ devices randomly selected as the monitors $(N=3,4, \cdots, 10$ in the $x$ axis), and for each $N$ we iterate the experiment 30 times. The vertical boxes at each $N$ shows the confidence interval of $\left(\mathbf{E}_{K} \mathcal{E}_{d B}^{2}\right)^{1 / 2}$ at $95 \%$ confidence, and the horizontal line connects the median value. It can be seen that the general trend as well as the median performance continue to improve as the number of cooperating monitors grows, and eventually reaches the point where even the worst case performance enters the effectiveness region (below $\sigma_{d B}=3.92$ ). This again demonstrates that the cooperation of monitors is a powerful tool that overcomes the inherent uncertainty in blind estimation of transmit power ${ }^{4}$.

\section{CONCLUSION}

We studied the problem of blind transmit-power estimation under wireless fading, wherein neither the transmitter location nor any statistical characterization of its transmit power are assumed. The setup consisted of a set of monitors that measured received power and the goal was to utilize these measurements to estimate the transmission power. We obtained analytical results for the Maximum Likelihood estimate and utilized the analysis to draw geometrical insights into the problem. We also showed asymptotic optimality of the estimate, wherein the estimate converges to the actual transmit power under certain conditions as the number of monitors increases. To understand the estimation error performance under the finite monitor case we presented

\footnotetext{
${ }^{4}$ Though not shown here due to space limitation, the performance of MLE-Coop-fmin and MLE-Pair show a trend similar to that in synthetic experiment in Section 5.4.
}

both synthetic simulation results and numerical analysis on a data-set of actual field measurements. Our work highlights a key aspect that even under no knowledge of the transmitter location and the statistical characteristics of its power, one can usefully exploit the spatial diversity in measurements through monitor cooperation to significantly enhance the transmit power estimation.

\section{ACKNOWLEDGEMENT}

The authors would like to thank Chatschik Bisdikian for helpful discussions on the topic.

Research was sponsored in part by the U.S. Army Research Laboratory and the U.K. Ministry of Defence and was accomplished under Agreement Number 911NF-06-3-0001. The views and conclusions contained in this document are those of the author(s) and should not be interpreted as representing the official policies, either expressed or implied, of the U.S. Army Research Laboratory, the U.S. Government, the U.K. Ministry of Defence or the U.K. Government. The U.S. and U.K. Governments are authorized to reproduce and distribute reprints for Government purposes notwithstanding any copyright notation hereon.

\section{REFERENCES}

[1] T. S. Rappaport, Wireless Communications: Principles and Practice, Englewood Cliffs, NJ: Prentice Hall, 1996.

[2] N. Patwari, J. Ash, S. Kyperountas, A. O. Hero, R. M. Moses and N. S. Correal, "Locating the Nodes: Cooperative Localization in Wireless Sensor Networks", IEEE Signal Processing Mag., vol. 22, no. 4, July 2005.

[3] C. Savarese, J. M. Rabaey and J. Beutel, "Locationing in distributed ad-hoc wireless sensor networks", in Proc. IEEE ICASSP, May 2001.

[4] R. L. Moses, D. Krishnamurthy, and R. Patterson, "A self-localization method for wireless sensor networks," EURASIP J. Applied Sig. Proc., no. 4, pp. 348-358, March 2003.

[5] E. G. Larsson, "Cramer-Rao bound analysis of distributed positioning in sensor networks," IEEE Signal Processing Letters,vol. 11, no. 3, March 2004.

[6] A. Dogandzic and B. Zhang, "Distributed estimation and detection for sensor networks using hidden markov random field models," IEEE Tran. on Signal Processing, vol. 54, no. 8, Aug. 2006.

[7] B. Krishnamachari and S. Iyengar, "Distributed Bayesian algorithms for fault-tolerant event region detection in wireless sensor networks," IEEE Trans. Comput., vol. 53, pp. 241-250, Mar. 2004.

[8] S. Kim, H. Jeon, H. Lee, and J. S. Ma, "Robust Transmission Power and Position Estimation in Cognitive Radio," International Conference on Information Networking(ICOIN'O'7), 2007.

[9] G. J. Foschini, "A simple distributed autonomous power control algorithm and its convergence", IEEE Tran. on Vehicular Tech., 42(4), 1993.

[10] S. Grandhi, J. Zander and R. Yates, "Constrained power control", International Journal of Wireless Personal Communications, 1(4), 1995.

[11] P. Viswanath, V. Anantharam, D. Tse, "Optimal sequences, power control and capacity of synchronous 
CDMA systems with linear MMSE multiuser receivers", IEEE Tran. Information Theory, vol. 45(6), 1999.

[12] I. W. Ho, B.-J. Ko, M. Zafer, C. Bisdikian, and K. Leung, "Cooperative transmit-power estimation in MANETs", Proc. IEEE WCNC 2008.

[13] A.J. Coulson, A.G. Williamson, and R.G. Vaughan, "A statistical basis for lognormal shadowing effects in multipath fading channels," IEEE Trans. Veh. Technol., vol. 46, no. 4, pp. 494-502, April 1998.

[14] N. Patwari, A.O. Hero III, M. Perkins, N.S. Correal, and R.J. O'Dea, "Relative location estimation in wireless sensor networks," IEEE Trans. Signal Processing, vol. 51, no. 8, pp. 2137-2148, Aug. 2003.

[15] H.L. Van Trees, Detection, Estimation, and Modulation Theory, Part I, New York: Wiley, 1968.

[16] G. R. Grimmett and D. R. Stirzaker, Probability and Random Processes, 3rd ed. New York, Oxford University Press, 2001.

[17] Wireless Sensor Network Localization Measurement Repository,http://www.eecs.umich.edu/ hero/localize/

\section{APPENDIX}

\section{A. PROOF OF THEOREM II}

Consider the joint density function $f(\mathbf{z} ; \hat{\theta})$ as given in (19). To maximize this function with respect to $\hat{\theta}$, it suffices to maximize the logarithm of $f(\mathbf{z} ; \hat{\theta})$. This gives,

$$
\hat{\theta}^{*}=\arg \min _{\hat{\theta}} \sum_{i=1}^{N}\left(z_{i}+\ln \left(d_{i}^{\alpha} / k\right)-Z\right)^{2}
$$

Let $J(Z, x, y)=\sum_{i=1}^{N}\left(z_{i}+\ln \left(d_{i}^{\alpha} / k\right)-Z\right)^{2}$ and consider its partial derivative with respect to $Z$. This gives,

$$
\frac{\partial J(Z, x, y)}{\partial Z}=-\sum_{i=1}^{N} 2\left(z_{i}+\ln \left(d_{i}^{\alpha} / k\right)-Z\right)
$$

For a fixed $(x, y)$, the optimal $Z$ that minimizes $J(Z, x, y)$ is obtained by setting the above partial derivative to zero. This gives a unique value of $Z$ as follows,

$$
Z=\ln (1 / k)+\frac{1}{N} \sum_{i=1}^{N} \ln \left(e^{z_{i}} d_{i}^{\alpha}\right)
$$

Thus, for any location $(x, y)$ the unique $Z$ that minimizes $J(Z, x, y)$ is as given above and the minimum value of $J(Z, x, y)$ at this location is $\tilde{J}(x, y)=\sum_{i=1}^{N}\left(\ln \left(e^{z_{i}} d_{i}^{\alpha}\right)-\frac{\sum_{j=1}^{N} \ln \left(e^{z_{j}} d_{j}^{\alpha}\right)}{N}\right)^{2}$ To obtain the global minimum we pick $\left(x^{*}, y^{*}\right)$ that minimizes $\tilde{J}(x, y)$ over $(x, y)$. Thus, the optimal solution $\left(Z^{*}, x^{*}, y^{*}\right)$ is as given in (21) and (22).

\section{B. PROOF OF LEMMA 1}

Using (3) we have $P_{i}=H_{i} \frac{k P}{d_{r, i}^{\alpha}}$, where $H_{i} \triangleq e^{W_{i}}$ is a Lognormal random variable. Substituting in (25) this gives, $P_{r}^{*}=$ $P\left(\prod_{i=1}^{N} H_{i}\right)^{1 / N}=P \tilde{H}_{N}$. Consider $\ln \left(\tilde{H}_{N}\right)=\frac{1}{N} \sum_{i=1}^{N} W_{i}$. Since $W_{i}$ are i.i.d Gaussian, by the Strong Law of large numbers [16] it converges almost surely to $\bar{W}=0$. Thus, $\tilde{H}_{N}$ converges almost surely to 1 .

\section{PROOF OF THEOREM IV}

Let $d_{i}^{*}$ denote the distance between monitor $i$ and location $\left(x_{N}^{*}, y_{N}^{*}\right)$ (the optimal solution for $(24)$ ) for an $N$ monitor scenario. Using (23), the power estimate $P_{N}^{*}$ is then given as, $\ln \left(P_{N}^{*}\right)=\ln \left(\frac{1}{k}\left(\prod_{i=1}^{N} p_{i}\left(d_{i}^{*}\right)^{\alpha}\right)^{1 / N}\right)$. From (3) we also have, $\ln \left(p_{i}\right)=\ln (k P)-\ln \left(\left(d_{r, i}\right)^{\alpha}\right)+w_{i}$, where $d_{r, i}$ is the actual distance between the transmitter and monitor $i$. Combining the above two we get, $\ln \left(P_{N}^{*}\right)=\ln (P)+\frac{\sum_{i=1}^{N} w_{i}}{N}+$ $\frac{\sum_{i=1}^{N} \alpha \ln \left(d_{i}^{*} / d_{r, i}\right)}{N}$. Taking limits we get,

$$
\lim _{N \rightarrow \infty} \ln \left(P_{N}^{*}\right)=\ln (P)+\lim _{N \rightarrow \infty} \frac{\sum_{i=1}^{N} \alpha \ln \left(d_{i}^{*} / d_{r, i}\right)}{N}
$$

where, by the Strong Law of Large Numbers (SLLN), almost surely, $\lim _{N \rightarrow \infty} \frac{\sum_{i=1}^{N} w_{i}}{N}=0$. Now, consider the objective function in (24) normalized by $N$. Denoting this normalized function as $J_{N}(\hat{x}, \hat{y})$ for a location $(\hat{x}, \hat{y}) \in \Gamma$, we have,

$$
J_{N}(\hat{x}, \hat{y})=\sum_{i=1}^{N} \frac{1}{N}\left(\ln \left(p_{i}\left(\hat{d}_{i}\right)^{\alpha}\right)-\frac{\sum_{j=1}^{N} \ln \left(p_{j}\left(\hat{d}_{j}\right)^{\alpha}\right)}{N}\right)^{2}
$$

Let $(x, y)$ be a generic location of a monitor drawn from the distribution $F_{X Y}(x, y)$. Let $L(\hat{x}, \hat{y})$ denote the random variable, $\alpha \ln \left(\frac{\hat{d}_{(x, y)}}{d_{r,(x, y)}}\right)$, where $\hat{d}_{(x, y)}$ is the distance from $(\hat{x}, \hat{y})$ to $(x, y)$ and $d_{r,(x, y)}$ is the distance from the transmitter location to $(x, y)$; thus $l_{i}=\alpha \ln \left(\frac{\hat{d}_{i}}{d_{r, i}}\right)$ is simply a particular realization of $L$ for monitor $i$. Using this, $J_{N}(\cdot)$ can be written as,

$$
\begin{aligned}
& J_{N}(\cdot)=\frac{1}{N} \sum_{i=1}^{N}\left(w_{i}-\frac{\sum_{j=1}^{N} w_{j}}{N}\right)^{2}+\frac{1}{N} \sum_{i=1}^{N}\left(l_{i}-\frac{\sum_{j=1}^{N} l_{j}}{N}\right)^{2} \\
& +2 \frac{1}{N} \sum_{i=1}^{N}\left(w_{i}-\frac{\sum_{j=1}^{N} w_{j}}{N}\right)\left(l_{i}-\frac{\sum_{j=1}^{N} l_{j}}{N}\right)
\end{aligned}
$$

If $(\hat{x}, \hat{y})=(0,0)$ (the transmitter location), then $l_{i}=0, \forall i, N$. Thus, by SLLN, $J_{N}(0,0) \rightarrow \sigma^{2}$ a.s. Suppose that $(\hat{x}, \hat{y})$ is not the transmitter location. From condition 1 in the Theorem statement, it implies that almost surely the monitors do not lie on an arc of a circle or a straight line; thus from Theorem III we have $L(\hat{x}, \hat{y}) \neq \beta$ (a constant), $\forall(\hat{x}, \hat{y})$, and the variance of $L(\hat{x}, \hat{y})$ is non-zero, i.e. $\operatorname{var}(L(\hat{x}, \hat{y}))>0$. Since, $w_{i}$ and $l_{i}$ are independent r.v's, by SLLN, we have $J_{N}(\hat{x}, \hat{y}) \rightarrow\left(\sigma^{2}+\operatorname{var}(L(\hat{x}, \hat{y}))\right)>\sigma^{2}, \forall(\hat{x}, \hat{y}) \neq(0,0)$ (note that the third term in (39) converges to $E[W] E[L]=0)$. Let $J_{N}^{*}$ denote the value of $(39)$ for $\left(x_{N}^{*}, y_{N}^{*}\right)$ (i.e. the minimizing value). From the above limits, we have $\lim _{N \rightarrow \infty} J_{N}^{*} \geq \sigma^{2}$. Also, $J_{N}^{*} \leq J_{N}(0,0), \forall N$ which gives $\lim _{N \rightarrow \infty} J_{N}^{*} \leq \sigma^{2}$. Thus, $\lim _{N \rightarrow \infty} J_{N}^{*}=\sigma^{2}$. Substituting in (39) and taking limits we get, $\frac{1}{N} \sum_{i=1}^{N}\left(l_{i}^{*}-\frac{\sum_{j=1}^{N} l_{j}^{*}}{N}\right)^{2} \rightarrow 0$. Since this is an average of positive terms, we require that all terms (except finitely many) must equal zero; equivalently in the limit $N \rightarrow \infty, l_{i}^{*}=\beta$ (a constant), $\forall i$ (except finitely many). However, from condition 1 in the theorem, almost surely, this cannot be true, except $\beta=0$ (corresponding to the actual transmitter location). Thus, $l_{N}^{*} \rightarrow 0$, which implies $\lim _{N \rightarrow \infty} \frac{\sum_{i=1}^{N} l_{i}^{*}}{N}=0$ (i.e. $\lim _{N \rightarrow \infty} \frac{\sum_{i=1}^{N} \alpha \log \left(d_{i}^{*} / d_{r, i}\right)}{N}=0$ ); combining with (37) we have the result. 